\title{
Effect of the on-period distribution on the performance of an ATM multiplexer fed by on/off sources : an analytical study
}

\author{
S. Wittevrongel and H. Bruneel \\ SMACS Research Group, Laboratory for Communications \\ Engineering, University of Ghent \\ Sint-Pietersnieuwstraat 41, B-9000 Gent, Belgium
}

\begin{abstract}
In this paper, we consider a statistical multiplexer in an ATM network, which is fed by a finite number of independent bursty on/off traffic sources. Most of the previous work assumes a two-state on/off source model, where both the on-periods and the off-periods are geometrically distributed. We assume a general distribution for the on-period lengths, and we study the effect of the on-period distribution on the multiplexer performance. Both the homogeneous and the heterogeneous traffic case are considered. An analytical method is presented to analyze the system, which basically is a generating-functions approach and uses an infinite-dimensional state description. Exact closed-form expressions are obtained for the mean and the tail distribution of the system contents. The numerical evaluation of the derived formulas is simple and not CPU time and/or memory space consuming, whatever the on-period distribution is. Numerical results indicate that, for a given fixed on-period length, the multiplexer performance strongly depends on the actual distribution of the on-period.
\end{abstract}

\section{Keywords}

ATM, multiplexer performance, on/off sources, general on-period distribution

\section{INTRODUCTION}

The ATM (Asynchronous Transfer Mode) is regarded as the most promising transfer technique for various types of information in future broadband integrated services digital networks. An ATM network is expected to support various services with widely different 
traffic characteristics, such as voice, data, video. Hence, for the design of these networks it is essential to assess the impact of the traffic characteristics on the performance.

In this paper, we consider a statistical multiplexer in an ATM network, which is modeled as a discrete-time single-server queueing system with infinite storage capacity. In ATM networks, time is divided into fixed-length slots and the transmission time of a cell is one slot. The multiplexer supports a finite number $N$ of independent bursty traffic sources. These sources belong to $T$ traffic types and for traffic type $t, 1 \leq t \leq T$, there are $N_{t}$ sources. Each source is modeled as an on/off source, that is, each source alternates between on-periods (active) and off-periods (passive). During an on-period, a source generates one cell per slot. No cells are generated during an off-period. We assume that for a source of type $t$, the length of the off-period is geometrically distributed with mean value $1 /\left(1-\beta_{t}\right)$. Furthermore, the lengths of the on-periods are assumed to be i.i.d. random variables with probability generating function (pgf) $A_{t}(z)$ and probability mass function (pmf) $a_{t}(i)$. Finally, it is assumed that the lengths of the on-periods and the off-periods are independently distributed.

The traffic model considered here allows us to study the impact of the on-period distribution on the multiplexer performance. Similar discrete-time models have been investigated in (Bruneel, 1988), (Steyaert, 1995) and (Xiong, 1992). In these papers homogeneous on/off sources $(T=1)$ were considered, and the on-periods were purely geometrically distributed, were distributed according to a mixture of 2 geometric distributions, or consisted of a geometrically distributed multiple of fixed-length intervals, respectively. The present paper can hence be viewed as an extension of these studies, in the sense that the distribution of the on-periods is general here. The study in this paper is also related to (Sohraby, 1993). However, the analysis presented there only leads to approximate results for the tail distribution of the system contents, whereas our analysis is exact and leads to closedform expressions for both the mean and the tail distribution (both coefficient and decay rate), whose numerical evaluation is not limited by the traffic characteristics. A general on-period distribution is also considered in (Elsayed, 1994). The system is analyzed there by numerically solving a set of balance equations, and hence the analysis is limited by the huge state space and the computational complexity of the algorithms. A heuristic approximation for the distribution of the system contents is derived in (Simonian, 1994).

The remainder of the paper is organized as follows. First, the homogeneous traffic case is considered. In Section 2, the analytical model of the multiplexer under study is described, and a functional equation is established which characterizes the behavior of the system under study. Section 3 concentrates on the steady-state cell arrival process. The mean and the tail distribution of the system contents are derived in Sections 4 and 5 respectively. In Section 6, the heterogeneous traffic case is considered. Some numerical examples are given in Section 7.

\section{SYSTEM EQUATIONS AND FUNCTIONAL EQUATION}

First, we will present the analysis for the case of a single traffic type (T=1), and for the clearness of the explanation, we omit the $t$-dependence in the above described source model.

As mentioned before, we assume that each source will alternately be passive (state 0 ), or active. An active source is in state $n, n \geq 1$, if it is in the $n$th slot of an on-period. Hence, each source can be characterized by an infinite-dimensional Markov chain with states $n, n \geq 0$, and 
transition probabilities as shown in Figure 1, where $p(n-1)$ is the probability of having an onperiod of at least $n$ slots, given that the on-period consists of at least $n-1$ slots, i.e.,

$p(n-1)=\left(1-\sum_{i=1}^{n-1} a(i)\right)\left(1-\sum_{i=1}^{n-2} a(i)\right)^{-1}$

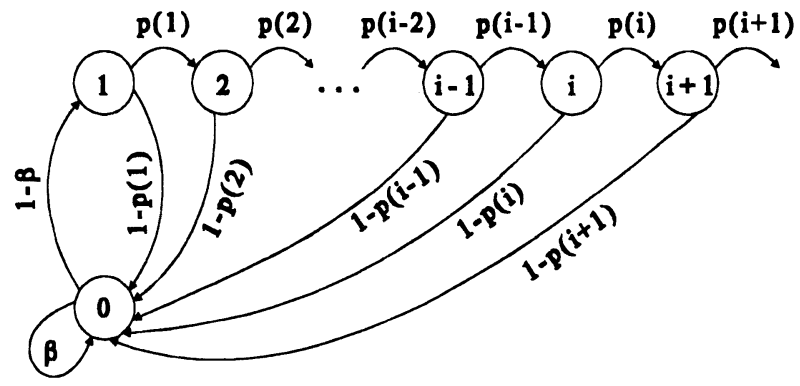

Figure 1 State transition diagram of an inlet.

Let us now define the random variables $d_{n, k}(n \geq 1)$ as the number of sources in the $n$th slot of an on-period during slot $k$. Then, in view of Figure 1, the following relationships hold :

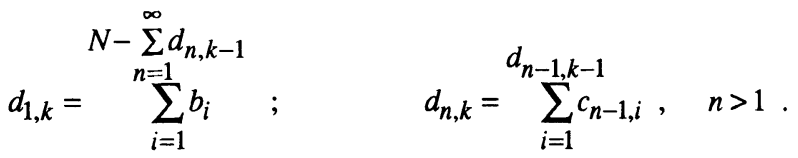

Here the $b_{i}$ 's are i.i.d. random variables with $\mathrm{pgf}$

$B(z) \triangleq E\left[z^{b_{i}}\right]=\beta+(1-\beta) z$.

For given $n$, the $c_{n-1, i}$ 's are i.i.d. random variables with $\mathrm{pgf}$

$C_{n-1}(z) \triangleq E\left[z^{c_{n-1, i}}\right]=1-p(n-1)+p(n-1) z, \quad n>1$.

Moreover, the $b_{i}$ 's and the $c_{n-1, i}$ 's are mutually independent. Also, let $e_{k}$ be the total number of cell arrivals during slot $k$. This random variable can be expressed as

$e_{k}=\sum_{n=1}^{\infty} d_{n, k}$ 
Next, let $s_{k}$ represent the system contents (i.e., the number of cells stored in the multiplexer buffer, including the potential cell in transmission) at the beginning of slot $k$, i.e., just after slot $(k-1)$. Then the evolution of the system contents is described by the following system equation,

$s_{k+1}=\left(s_{k}-1\right)^{+}+e_{k}$

where (.)+ denotes $\max (., 0)$. Equations (1)-(6) imply that the set $\left\{\left(d_{n, k-1}(n \geq 1), s_{k}\right)\right\}$ is a Markov chain. If we now define the joint pgf of $d_{n, k-1}(n \geq 1)$ and $s_{k}$ as

$P_{k}\left(x_{1}, x_{2}, \ldots, z\right)=E\left[\left(\prod_{n=1}^{\infty} x_{n}^{d_{n, k-1}}\right) s^{s_{k}}\right]$

and if we assume that the queueing system can reach a steady state, by using equations (2)-(6), in a similar way as described e.g. in (Xiong, 1992), we obtain the following functional equation for the steady-state version $P\left(x_{1}, x_{2}, \ldots, z\right)$ of $P_{k}\left(x_{1}, x_{2}, \ldots, z\right)$ :

$P\left(x_{1}, x_{2}, \ldots, z\right)=\frac{\left[B\left(x_{1} z\right)\right]^{N}}{z}\left\{P\left(\frac{C_{1}\left(x_{2} z\right)}{B\left(x_{1} z\right)}, \frac{C_{2}\left(x_{3} z\right)}{B\left(x_{1} z\right)}, \ldots, z\right)+(z-1) p_{0}\right\}$,

where the quantity $p_{0}$ indicates the probability of having an empty buffer at the beginning of an arbitrary slot in the steady state.

Next, let $s$ be the system contents at the beginning of a slot in the steady state. Unfortunately, we are not able to derive from (8) an explicit expression for $P\left(x_{1}, x_{2}, \ldots, z\right)$ or not even for the pgf $S(z)$ of $s$. However, as shown in the following, it is possible to derive the moments and the tail distribution of $\mathrm{s}$, if we now consider in (8) only those values of $x_{n}(n \geq 1)$ and $z$ for which the arguments of the $P$-functions on both sides of (8) are equal to each other, i.e., $x_{n}=C_{n}\left(x_{n+1} z\right) / B\left(x_{1} z\right), n \geq 1$. From this equation, $x_{n}(n \geq 1)$ can be solved in terms of $z$. It turns out that for a given value of $z$, there may be more than one set of solutions. Here, we only choose the set of solutions which has the additional property that $x_{n}=1, n \geq 1$, for $z=1$. Denoting this set of solutions by $\chi_{n}(z)$, we get

$z \chi_{n}(z)=\left\{\sum_{i=n}^{\infty} a(i)\left(\frac{B\left(\chi_{1}(z) z\right)}{z}\right)^{n-1-i}\right\}\left\{1-\sum_{i=1}^{n-1} a(i)\right\}^{-1}, \quad n \geq 1$.

Note in particular that

$z \chi_{1}(z)=A\left(\frac{z}{\beta+(1-\beta) \chi_{1}(z) z}\right)$.

By choosing $x_{n}=\chi_{n}(z), n \geq 1$, in (10), we then obtain the function $P\left(\chi_{1}(z), \chi_{2}(z), \ldots, z\right)$ as 
$P\left(\chi_{1}(z), \chi_{2}(z), \ldots, z\right)=\frac{(z-1) p_{0} G(z)}{z-G(z)}$,

where

$G(z)=\left[B\left(\chi_{1}(z) z\right)\right]^{N}=\left[\beta+(1-\beta) \chi_{1}(z) z\right]^{N}$.

From the normalization condition $\left.P\left(\chi_{1}(z), \chi_{2}(z), \ldots, z\right)\right|_{z=1}$, it follows that $p_{0}=1-p$, where $p$ is the total load into the multiplexer, i.e.,

$p=N A^{\prime}(1)(1-\beta) /\left[1+A^{\prime}(1)(1-\beta)\right]$.

In the next sections, we will describe a technique to calculate exactly the moments and the tail distribution of the buffer occupancy without having to calculate the whole distribution of $s$.

\section{THE CELL ARRIVAL PROCESS}

In this section, we will derive the steady-state pgf of the cell arrival process. Let $d_{n}$ denote the number of sources in the $n$th slot of an on-period during an arbitrary slot in the steady state. The joint pgf $D\left(x_{1}, x_{2}, \ldots\right)$ of the random variables $d_{n}(n \geq 1)$ is given by $P\left(x_{1}, x_{2}, \ldots, 1\right)$. Putting $z=1$ in the functional equation (8), we get

$D\left(x_{1}, x_{2}, \ldots\right)=\left[B\left(x_{1}\right)\right]^{N} D\left(\frac{C_{1}\left(x_{2}\right)}{B\left(x_{1}\right)}, \frac{C_{2}\left(x_{3}\right)}{B\left(x_{1}\right)}, \ldots\right)$

$D\left(x_{1}, x_{2}, \ldots\right)$ is an $N$ th degree polynomial in $x_{n}, n \geq 1$, and it can be verified that the above equation is satisfied if

$D\left(x_{1}, x_{2}, \ldots\right)=(1-\sigma)^{N}\left[1+(1-\beta) \sum_{n=1}^{\infty} \sum_{i=n}^{\infty} a(i) x_{n}\right]^{N}$,

where $\sigma$ is the load of one source, i.e., $\sigma=p / N$. The marginal $\operatorname{pgf} D_{n}(z)$ of $d_{n}$ is then obtained by putting $x_{i}=1(i \geq 1, i \neq n)$ and $x_{n}=z$ in (15). The mean value of $d_{n}$ is given by

$$
E\left[d_{n}\right]=D_{n}^{\prime}(1)=\frac{N(1-\beta)}{1+(1-\beta) A^{\prime}(1)}\left(1-\sum_{i=1}^{n-1} a(i)\right),
$$

i.e., the mean number of sources in the first slot of an on-period during a slot times the probability of having an on-period of at least $n$ slots. From (16), the mean number of arrivals during a slot is found to be equal to $p$. Consequently, the equilibrium condition is $p<1$. 


\section{MEAN SYSTEM CONTENTS}

In this section, we derive an expression for the mean buffer occupancy $E[s]$. First, we define the burstiness factor $K$ of the source as

$K=A^{\prime}(1)(1-\sigma)=\frac{\sigma}{1-\beta}$,

where $1 /(1-\beta)$ is the mean off-period length and $\sigma$ is the average load of a source. Note that $K$ equals the ratio of the mean on-period length in our model, to the mean length of an on-period in case of a Bernoulli arrival process. It is clear that $\sigma$ describes the ratio of the mean lengths of the on/off periods, whereas $K$ is a measure for the absolute lengths of these periods. Also we define the variance factor $L$ of the source as the ratio of the variance of the on-period length in our model, to the variance of a geometrically distributed on-period with the same mean length, i.e.,

$L=\frac{A^{\prime \prime}(1)+A^{\prime}(1)-\left[A^{\prime}(1)\right]^{2}}{A^{\prime}(1)\left[A^{\prime}(1)-1\right]}$.

Next, by evaluating the first derivative of equation (11) with respect to $z$ at $z=1$, in a similar way as explained in (Bruneel, 1988), we get

$E[s]=p+\frac{(N-1) p^{2}}{2 N(1-p)}\left[K+L(K-1)+\frac{p}{N}(L-1)\right]$.

It has been checked that the above general result is in agreement with the results obtained in (Bruneel, 1988), (Steyaert, 1995) and (Xiong, 1992). The above formula clearly demonstrates that the multiplexer performance depends not only on the mean length of the on-period, but also strongly on the actual on-period-length distribution. First of all, we observe that for a given total load, the mean length of the on-period has a substantial influence on $E[s]$. The mean system contents namely linearly increases with the burstiness factor $K$ of the sources. Next, for a given load and a given mean length of the on-period (given $K$ ), the mean system contents $E[s]$ linearly increases with $L$, i.e., $E[s]$ increases linearly with the variance of the onperiod. Higher-order moments of the on-period distribution have no impact on the mean system contents.

\section{TAIL DISTRIBUTION OF THE SYSTEM CONTENTS}

From the inversion formula for z-transforms it follows that the pmf Prob $[s=n]$ of $s$ can be expressed as a weighted sum of negative powers of the poles of $S(z)$. Since the modulus of all these poles is larger than one, it is obvious that for large $n$, Prob $[s=n]$ is dominated by the contribution of the pole having the smallest modulus. Let us denote this dominating pole by $z_{0}$. It is shown in (Bruneel, 1994) that in order to ensure that the tail distribution is nonnegative 
anywhere, $z_{0}$ must necessarily be real and positive. Furthermore, we assume here that $z_{0}$ has multiplicity one. Therefore, for $n$ sufficiently large, $\operatorname{Prob}[s=n]$ can be approximated as

$\operatorname{Prob}[s=n] \cong-\frac{\theta}{z_{0}}\left(\frac{1}{z_{0}}\right)^{n}$

where $\theta$ is the residue of $S(z)$ in the point $z=z_{0}$.

\subsection{Calculation of $z_{0}$}

As in (Xiong, 1992), it can be argued that $z_{0}$ is also the pole with the smallest modulus of $P\left(\chi_{1}(z), \chi_{2}(z), \ldots, z\right)$. Hence, in view of (11) and (12), $z_{0}$ is a real root of $z-G(z)=0$, or

$z-\left[\beta+(1-\beta) \chi_{1}(z) z\right]^{N}=0$

This can even be proved. As all sources are statistically independent, $G(z)$ is the PerronFrobenius eigenvalue related to the aggregated arrival process to the multiplexer (Neuts, 1989). Hence, the dominant pole $z_{0}$ of $S(z)$ is determined by $z-G(z)=0$ (Sohraby, 1993). It is obvious that $\chi_{1}(z)>0$ for $z>1$. From (10) and (21), we have

$\frac{z^{1 / N}-\beta}{1-\beta}-A\left(\frac{z}{z^{1 / N}}\right)=0$.

Hence, the pole $z_{0}$ can be easily calculated exactly from equation (22) by using, for instance, the Newton-Raphson algorithm.

Next, in order to assess the impact of the traffic characteristics on the geometric decay rate, we derive 2 approximations for $z_{0}$ in the heavy traffic case, where the total utilization of the multiplexer approaches to one. In case of heavy traffic, it is expected that $z_{0}$ will be close to one. By expanding the equation $z=G(z)$ around $z=1$, we obtain

$z=G(1)+G^{\prime}(1)(z-1)+\frac{G^{\prime \prime}(1)}{2}(z-1)^{2}+\frac{G^{\prime \prime \prime}(1)}{6}(z-1)^{3}+O(z-1)^{4}$.

By keeping terms up to $(z-1)^{2}$ in equation (23) and neglecting higher-order terms, we get the following approximation for $z_{0}$ :

$z_{0} \cong z_{1} \triangleq 1+\frac{2(1-p)}{p(p-1)+p\left(1-\frac{p}{N}\right)\left[K+L(K-1)+\frac{p}{N}(L-1)\right]}$.

A more accurate heavy-traffic approximation for $z_{0}$ is obtained by keeping terms up to $(z-1)^{3}$ in equation (23) and neglecting higher-order $(\geq 4)$ terms. As a result, we find 
$z_{0} \cong z_{2} \triangleq 1+\frac{-3 G^{\prime \prime}(1)+3 \sqrt{\left[G^{\prime \prime}(1)\right]^{2}+\frac{8}{3}(1-p) G^{\prime \prime \prime}(1)}}{2 G^{\prime \prime \prime}(1)}$,

where

$$
\begin{aligned}
G^{\prime \prime}(1)= & N(N-1) \sigma^{2}+N \sigma(1-\sigma)[(L+1)(K+\sigma-1)-2 \sigma] ; \\
G^{\prime \prime \prime}(1)= & N(N-1)(N-2) \sigma^{3}+3 N(N-1) \sigma^{2}[(1-\sigma)(L+1)(K+\sigma-1)-2 \sigma(1-\sigma)] \\
& +N \sigma(1-\sigma)\left[-3 \sigma(L+1)^{2}(K+\sigma-1)^{2}+12 \sigma^{2}(1-\sigma)-(1-\sigma)^{2}+(1-\sigma)^{3} \frac{M}{K}\right] \\
& +N \sigma(1-\sigma)(L+1)(K+\sigma-1) 3\left(4 \sigma^{2}-2 \sigma-1\right),
\end{aligned}
$$

and $M$ is the third moment of the on-period distribution, i.e., $M=A^{\prime \prime \prime}(1)+3 A^{\prime \prime}(1)+A^{\prime}(1)$. In Table 1 , we compare the exact value of $z_{0}$ with the approximations $z_{1}$ and $z_{2}$, for $N=8$, a negative binomial distribution for the on-period length, i.e., $A(z)=(1-\gamma)^{2} z /(1-\gamma z)^{2}$ and various values of the load $p$ and the burstiness factor $K$. The results show that both approximations are accurate for very high values of $p$, and behave in the same way as the pole $z_{0}$ as the source characteristics vary. Moreover $z_{2}$ is also quite accurate for intermediate loads. Expression (24) indicates that the variance of the on-period length has a strong influence on the heavy-traffic tail behavior of the multiplexer. Furthermore, as the total utilization approaches to one, the geometric decay rate $1 / z_{0}$ becomes almost independent of higher-order $(\geq 3)$ moments of the on-period distribution. For intermediate to high values of the load however, an accurate performance evaluation can be obtained by taking into account the first 3 moments of the onperiod distribution. Studies based only on the first 2 moments could in this case lead to inaccurate results, and should be applied with careful consideration.

Table 1 Exact and approximate results for the dominant pole $z_{0}$, for $N=8$ and a negative binomial distribution for the on-period length

\begin{tabular}{lllll}
\hline$p$ & $K$ & $z_{0}$ & $z_{1}$ & $z_{2}$ \\
\hline 0.6 & 2 & 1.435059 & 1.632583 & 1.463659 \\
0.7 & 2 & 1.305059 & 1.395415 & 1.315785 \\
0.8 & 2 & 1.191038 & 1.224442 & 1.193924 \\
0.9 & 2 & 1.090056 & 1.097130 & 1.090392 \\
0.6 & 10 & 1.067622 & 1.099755 & 1.072289 \\
0.7 & 10 & 1.049399 & 1.064594 & 1.051180 \\
0.8 & 10 & 1.032153 & 1.037955 & 1.032641 \\
0.9 & 10 & 1.015725 & 1.016992 & 1.015783 \\
\hline
\end{tabular}




\subsection{Calculation of $\theta$}

Let us consider the case where the number of cells stored in the multiplexer buffer just after a given slot is sufficiently large $(\gg N)$. Then we may think that the number of cell arrivals during this slot (which cannot be larger than $N$ ) has almost no impact on the total buffer contents. Consequently, if $n$ is sufficiently large $(n>T)$, we may assume that the conditional probabilities $\operatorname{Prob}\left[d_{1}=i_{1}, d_{2}=i_{2}, \ldots \mid s=n\right]$ are almost independent of $n$, and approach to some limiting values for $n \rightarrow \infty$, denoted by $\omega\left(i_{1}, i_{2}, \ldots\right)$, i.e.,

$\operatorname{Prob}\left[d_{1}=i_{1}, d_{2}=i_{2}, \ldots \mid s=n\right] \cong \omega\left(i_{1}, i_{2}, \ldots\right), \quad n>T$,

with corresponding joint pgf $\Omega\left(x_{1}, x_{2}, \ldots\right)$.

Using equation (26), the joint pgf $P\left(x_{1}, x_{2}, \ldots, z\right)$ can now be approximately expressed as

$$
\begin{aligned}
P\left(x_{1}, x_{2}, \ldots, z\right) \cong & \sum_{i_{1}} \sum_{i_{2}} \ldots \sum_{j=0}^{T} \operatorname{Prob}\left[d_{1}=i_{1}, d_{2}=i_{2}, \ldots, s=j\right]\left(\prod_{n=1}^{\infty} x_{n}^{i_{n}}\right) z^{j} \\
& +\Omega\left(x_{1}, x_{2} \ldots\right)\left(S(z)-\sum_{j=0}^{T} \operatorname{Prob}[s=j] z^{j}\right) .
\end{aligned}
$$

Setting $x_{n}=\chi_{n}(z)$, we know that $z_{0}$ is a pole of both the $P$-function and $S(z)$. As $T$ is finite, multiplying both sides of the above equation by $\left(z-z_{0}\right)$ and taking the $z \rightarrow z_{0}$ limit, we find

$\theta=\frac{\left(z_{0}-1\right)(1-p) z_{0}}{\left[1-G^{\prime}\left(z_{0}\right)\right] \Omega\left(\chi_{1}\left(z_{0}\right), \chi_{2}\left(z_{0}\right), \ldots\right)}$

In order to derive the $\operatorname{pgf} \Omega\left(x_{1}, x_{2}, \ldots\right)$, we let $\pi\left(i_{1}, i_{2}, \ldots \mid j_{1}, j_{2}, \ldots\right)$ denote the one-step transition probability that there are $i_{n}(n \geq 1)$ sources in the $n$th slot of an on-period, given that there were $j_{l}(l \geq 1)$ sources in the $l$ th slot of an on-period in the previous slot. From equations (20) and (26), we then get

$z_{0} \omega\left(i_{1}, i_{2}, \ldots\right)=\sum_{j_{1}} \sum_{j_{2}} \ldots \pi\left(i_{1}, i_{2}, \ldots \mid j_{1}, j_{2}, \ldots\right) \omega\left(j_{1}, j_{2}, \ldots\right)\left(\mathrm{z}_{0}\right)^{\sum_{k=1}^{\infty} i_{k}}$

Hence, we obtain the following equation for the $\operatorname{pgf} \Omega\left(x_{1}, x_{2}, \ldots\right)$ :

$z_{0} \Omega\left(x_{1}, x_{2}, \ldots\right)=\left[B\left(x_{1} z_{0}\right)\right]^{N} \Omega\left(\frac{C_{1}\left(x_{2} z_{0}\right)}{B\left(x_{1} z_{0}\right)}, \frac{C_{2}\left(x_{3} z_{0}\right)}{B\left(x_{1} z_{0}\right)}, \ldots\right)$ 
As can be expected intuitively, it is possible to show that the solution $\Omega\left(x_{1}, x_{2}, \ldots\right)$ of $(28)$ has the same form of expression as the $\operatorname{pgf} D\left(x_{1}, x_{2}, \ldots\right)$ of the unconditional cell arrival process. Specifically, $\Omega\left(x_{1}, x_{2}, \ldots\right)$ can be expressed as

$$
\Omega\left(x_{1}, x_{2}, \ldots\right)=\left(1-\sum_{n=1}^{\infty} \sigma_{n}^{*}+\sum_{n=1}^{\infty} \sigma_{n}^{*} x_{n}\right)^{N},
$$

where $\sigma_{n}^{*}(n \geq 1)$ is the (conditional) probability of finding a source in the $n$th slot of an onperiod, when the number of cells in the buffer is extremely large. From equations (9), (21), (28) and (29), an expression can be derived for $\Omega\left(\chi_{1}\left(z_{0}\right), \chi_{2}\left(z_{0}\right), \ldots\right)$. Also, from equations (10) and (12), we obtain an expression for $G^{\prime}\left(z_{0}\right)$. Finally, after some algebra, we find

$$
\theta=\frac{(1-p) z_{0}^{2}\left(z_{0}-1\right)^{N+1}}{\left[1-A^{\prime}\left(\frac{z_{0}}{z_{0}^{1 / N}}\right) \frac{(N-1)(1-\beta) z_{0}}{z_{0}^{2 / N}}\right]\left[A^{\prime}\left(\frac{z_{0}}{z_{0}^{1 / N}}\right) \frac{(1-\beta) z_{0}}{z_{0}^{2 / N}}+1\right]^{N-1}\left(z_{0}-z_{0}^{1 / N}\right)^{N}} .
$$

Consequently, the two parameters $z_{0}$ and $\theta$ of the geometric tail approximation have been determined. It is then easy to calculate the probability $\operatorname{Prob}[s>S]$ that the buffer contents exceeds a certain threshold $S$, which is often used in practice to approximate the cell loss ratio in a finite buffer with a waiting room of size $S$, i.e., the fraction of the arriving cells that is lost upon arrival because of buffer overflow.

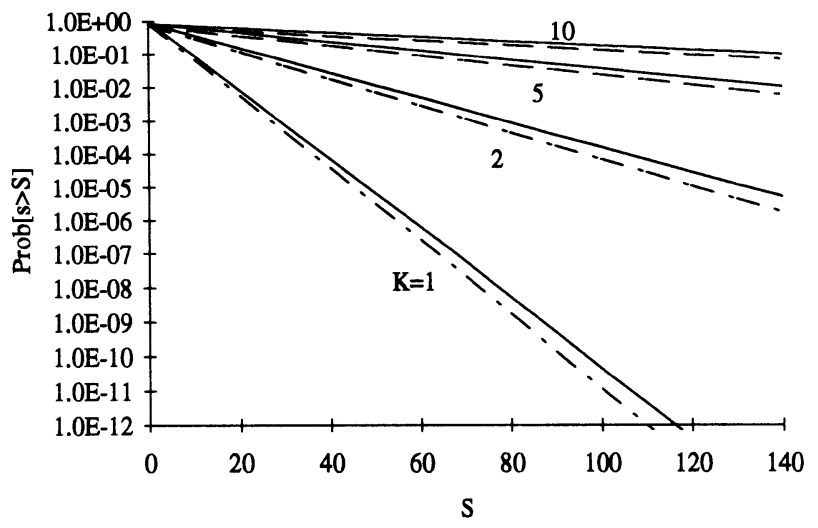

Figure 2 Prob $[s>S]$ versus $S$ : exact results (solid lines) and heavy-traffic approximation (dashed lines), for $N=8, \mathrm{p}=0.9$, and a negative binomial distribution for the on-period length. 
In Figure 2, we compare Prob[ $s>S]$ calculated from (22) and (30), with the heavy-traffic approximation calculated from (24) and (30), for $N=8, p=0.9$, a negative binomial distribution for the on-period length and various values of $K$. We see that for $p=0.9$, the heavy-traffic approximation based on the first 2 moments of the on-period distribution is quite accurate, and somewhat overestimates $\operatorname{Prob}[s>S]$. Hence, we may conclude, that for very high utilization, the "overflow probability" is nearly independent of higher-order $(\geq 3)$ moments of the onperiod length.

\section{THE HETEROGENEOUS TRAFFIC CASE}

In this section, we consider the case of heterogeneous traffic sources. We assume that there are $T$ traffic types, and for traffic type $t$, there are $N_{t}$ sources. The mean and the tail distribution of the system contents can then be derived in a similar way as described above for the homogeneous traffic case. Specifically, the mean system contents $E[s]$ is given by

$$
E[s]=p+\frac{1}{2(1-p)} \sum_{t=1}^{T} N_{t} \sigma_{t}\left(p-\sigma_{t}\right)\left[K_{t}+L_{t}\left(K_{t}-1\right)+\sigma_{t}\left(L_{t}-1\right)\right]
$$

Here $p$ is the total load into the multiplexer and $\sigma_{t}, K_{t}$ and $L_{t}$ denote the load, the burstiness factor and the variance factor respectively, of a source of traffic type $t$. The dominant pole $z_{0}$ of $S(z)$ is determined by the set of equations

$$
z-\prod_{t=1}^{T}\left[\beta_{t}+\left(1-\beta_{t}\right) \chi_{1, t}(z) z\right]^{N_{t}}=0 ; \quad z \chi_{1, t}(z)=A_{t}\left(\frac{z}{\beta_{t}+\left(1-\beta_{t}\right) \chi_{1, t}(z) z}\right),
$$

and hence $z_{0}$ can easily be obtained by means of the Newton-Raphson algorithm. The residue $\theta$ is given by

$$
\theta=(1-p) z_{0}^{2}\left(z_{0}-1\right)^{N+1}\left(1-\sum_{t=1}^{T} \frac{N_{t} F_{t}\left(z_{0}\right)}{1+F_{t}\left(z_{0}\right)}\right)^{-1} \prod_{t=1}^{T}\left[\left(z_{0}-\left(z_{0}(t)\right)^{1 / N_{t}}\right)\left(1+F_{t}\left(z_{0}\right)\right)\right]^{-N_{t}},
$$

where

$$
z_{0}(t)=\left[\beta_{t}+\left(1-\beta_{t}\right) \chi_{1, t}\left(z_{0}\right) z_{0}\right]^{N_{t}} ; \quad F_{t}\left(z_{0}\right)=\left(1-\beta_{t}\right) z_{0}\left(z_{0}(t)\right)^{-2 / N_{t}} A_{t}^{\prime}\left(\frac{z_{0}}{\left(z_{0}(t)\right)^{1 / N_{t}}}\right)
$$

\section{NUMERICAL EXAMPLES}

We will now illustrate the above analysis by means of some numerical examples. In order to show more clearly the influence of various traffic parameters of the sources on the multiplexer performance, let us first consider the case of a single traffic type. In Figure 3, we have plotted 
the mean system contents $E[s]$ in terms of the total load $p=N \sigma$, for $N=16, K=5$, and various values of the variance factor $L$ of the sources. The figure shows that for given values of $p$ and $K$, the variance of the on-period lengths strongly influences the mean system contents. The figure also indicates a considerable decrease in performance as $L$ increases.

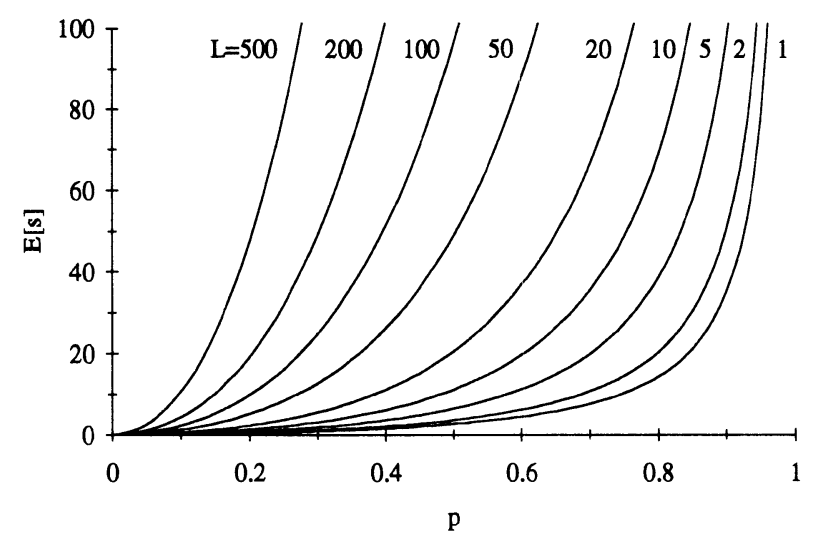

Figure 3 Mean system contents $E[s]$ versus the load $p$, for $N=16$ and $K=5$.

Next, we consider the following examples for the pgf $A(z)$ :

$A_{1}(z)=z^{m} ; \quad A_{2}(z)=\frac{(1-\gamma)^{2} z}{(1-\gamma z)^{2}} ; \quad A_{3}(z)=\frac{(1-\alpha) z}{1-\alpha z}$

i.e. constant-length on-periods, a negative binomial distribution and a geometric distribution respectively. In order to study the impact of the variance of the on-periods on the "overflow probability" Prob $[s>S]$, we choose the parameters of these distributions such that the mean on-period length $A^{\prime}(1)$ is equal to $m$ in all cases, which corresponds to choosing

$\alpha=\frac{m-1}{m}$ and $\gamma=\frac{m-1}{m+1}$.

The corresponding variances of the on-period lengths are then

$\operatorname{var}_{1}=0 ; \quad \operatorname{var}_{2}=\frac{1}{2}(m-1)(m+1) ; \quad \operatorname{var}_{3}=m(m-1)$.

In Figure 4, $\operatorname{Prob}[s>S]$ is plotted versus $S$, for $N=16, p=0.8, K=5$ and the above 3 distributions for the length of the on-period. The corresponding variances of the on-period lengths are then $\operatorname{var}_{1}=0, \operatorname{var}_{2}=13.35$ and $\operatorname{var}_{3}=22.44$. The variance factors are given by $L_{1}=0$, $L_{2}=0.595$ and $L_{3}=1$. It is clear that for given values of $p$ and $m$, the variance of the on-periods has a strong impact on the performance. We observe that the performance degrades with increasing variance of the on-period lengths. 


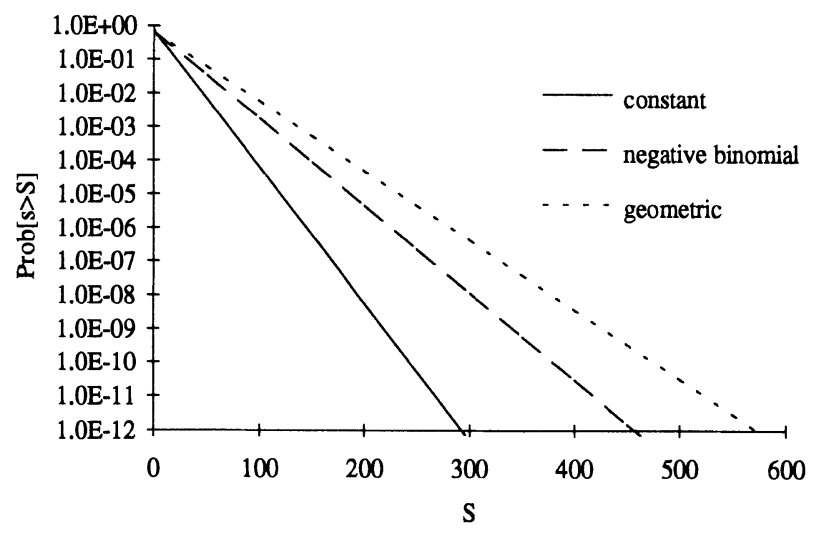

Figure 4 Prob $[\mathrm{s}>S]$ versus $S$, for $N=16, p=0.8, K=5$, and various on-period distributions.

Now, we consider a mixture of 2 geometric distributions for the on-period length, i.e.,

$A(z)=\frac{q\left(1-\alpha_{1}\right) z}{1-\alpha_{1} z}+\frac{(1-q)\left(1-\alpha_{2}\right) z}{1-\alpha_{2} z}$.

In Figure 5, we have plotted Prob[s>S] in terms of $S$, for $N=8, p=0.8, K=5, L=2$ and various values of $q$. We see that in general, Prob $[s>S]$ is not only determined by $p, K$ and $L$, but also depends on higher-order moments of the on-period-length distribution. Therefore, as mentioned before in Section 5.1, the commonly used assumption that it suffices to take into account only the first 2 moments of the on-period distribution should be used with care.

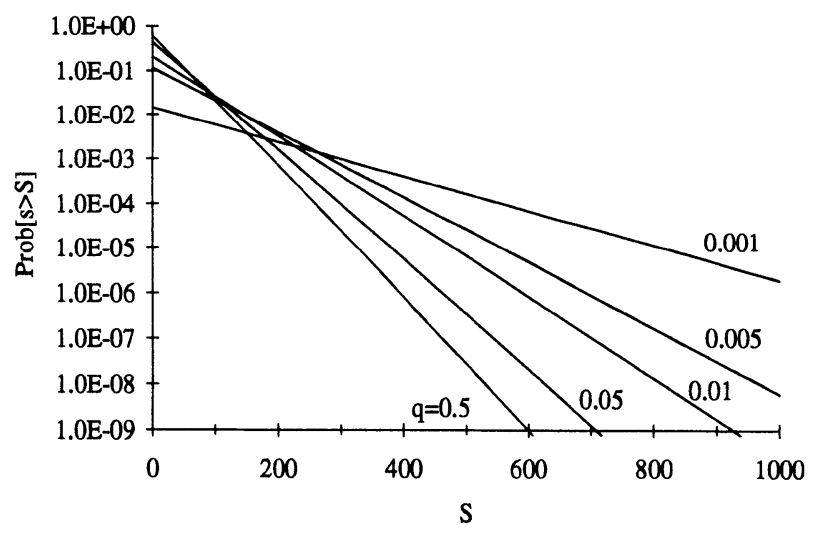

Figure 5 Prob $[s>S]$ versus $S$, for $N=8, p=0.8$, a mixture of 2 geometric distributions for the length of the on-period, $K=5, L=2$ and various values of $q$. 


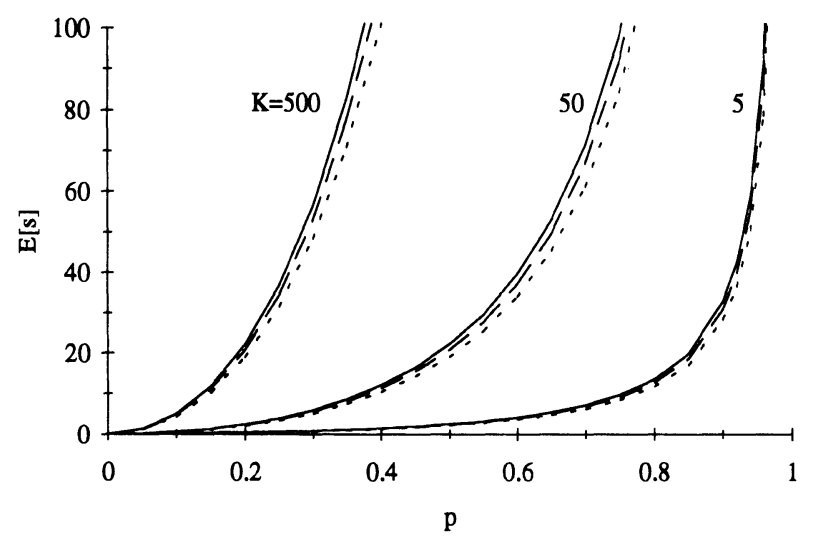

Figure 6 Mean system contents $E[s]$ versus the load $p$, for $T=2, N_{1}=N_{2}=4, K_{1}=K_{2}=K$, a geometric distribution for the on-period length, $\sigma_{1}=a \sigma_{2}, K=5,50,500$ and $a=0$ (dotted lines), $a=0.2$ (dashed lines), $a=1$ (solid lines).

Finally, we consider a multiplexer fed by 2 types of traffic sources, where $N_{1}=N_{2}=4$, $K_{1}=K_{2}=K$ and the on-periods are geometrically distributed. In order to assess the impact of the heterogeneity of the loads of these traffic types, we consider the case that $\sigma_{1}=a \sigma_{2}$, for various values of $a$. In Figure 6, we have plotted $E[s]$ as a function of $p$, for various values of $K$ and $a$. First of all, the figure reveals very clearly the strong impact of the burstiness factor $K$ on $E[s]$, for all values of $p$. Hence, the influence of the absolute lengths of the on-periods and the offperiods on $E[s]$ is far from negligible, even when the ratio of these lengths is fixed. The performance deteriorates considerably as $K$ increases. Secondly, for a given value of $K, E[s]$ decreases as the difference between $\sigma_{1}$ and $\sigma_{2}$ increases. This is also intuitively clear. The extreme cases are $a=1$, i.e., 8 homogeneous sources each with load $p / 8$, and $a=0$, i.e., 4 homogeneous sources each with load $p / 4$. Since for $a=1$, a maximum number of 8 cells can arrive during the same slot, whereas for $a=0$, this number is limited to 4 , it is expected that the case $a=1$ will lead to a higher $E[s]$. However, the impact of the heterogeneity of the loads is limited as compared to the strong influence of the burstiness of the sources.

\section{ACKNOWLEDGEMENT}

The authors wish to thank the Belgian National Fund for Scientific Research (N.F.W.O.) for support of this research.

\section{REFERENCES}

Bruneel, H. (1988) Queueing behavior of statistical multiplexers with correlated inputs. IEEE Transactions on Communications, 36, 1339-1341. 
Bruneel, H. and Kim, B. G. (1993) Discrete-time models for communication systems including ATM. Kluwer Academic Publishers, Boston.

Bruneel, H.; Steyaert, B.; Desmet, E. and Petit, G. (1994) Analytic derivation of tail probabilities for queue lengths and waiting times in ATM multiserver queues. European Journal of Operational Research, 76, 563-572.

Elsayed, K. (1994) On the superposition of discrete-time Markov renewal processes and application to statistical multiplexing of bursty traffic sources. Proceedings of IEEE GLOBECOM '94, San Francisco, 1113-1117.

Neuts, M. (1989) Structured stochastic matrices of M/G/1 type and their applications. Marcel Dekker Inc., New York.

Simonian, A. and Guibert, J. (1994) Large deviations approximation for fluid queues fed by a large number of on/off sources. Proceedings of ITC 14, Antibes Juan-les-Pins, 1013-1022.

Sohraby, K. (1993) On the theory of general ON-OFF sources with applications in high-speed networks. Proceedings of IEEE INFOCOM '93, San Francisco, 401-410.

Steyaert, B. and Bruneel, H. (1995) On the performance of multiplexers with three-state bursty sources : analytical results. IEEE Transactions on Communications, 43, 1299-1303.

Xiong, Y. and Bruneel, H. (1992) Performance of statistical multiplexers with finite number of inputs and train arrivals. Proceedings of IEEE INFOCOM '92, Firenze, 2036-2044.

\section{BIOGRAPHIES}

Sabine WITTEVRONGEL was born in Gent, Belgium, in 1969. She received the M.S. degree in Electrical Engineering from the University of Ghent, Belgium, in 1992. Since September 1992, she has been with the SMACS Research Group, Laboratory for Communications Engineering, University of Ghent, first in the framework of various projects, and since October 1994, as a researcher for the Belgian National Fund for Scientific Research (N.F.W.O.). Her main research interests include discrete-time queueing theory, performance evaluation of ATM networks and the study of traffic control mechanisms.

Herwig BRUNEEL was born in Zottegem, Belgium, in 1954. He received the M.S. degree in Electrical Engineering, the degree of Licentiate in Computer Science, and the Ph.D. degree in Computer Science in 1978, 1979 and 1984 respectively, all from the University of Ghent, Belgium. Since 1979, he has been working as a researcher for the Belgian National Fund for Scientific Research (N.F.W.O.) at the University of Ghent, where he currently leads the SMACS Research Group of the Laboratory for Communications Engineering. He is also a Professor in the Faculty of Applied Sciences at the same university. His main research interests include stochastic modeling of digital communication systems, discrete-time queueing theory, and the study of ARQ protocols. He has published more than 80 papers on these subjects and is coauthor of the book H. Bruneel and B. G. Kim, "Discrete-Time Models for Communication Systems Including ATM" (Kluwer Academic Publishers, Boston, 1993). 